\title{
TV/Series
}

$2 \mid 2012$

Les séries télévisées dans le monde : Échanges, déplacements et transpositions

\section{La localisation à l'étranger dans les séries télévisuelles contemporaines et l'affirmation internationale de la Corée du Sud}

\section{Stéphane Thévenet}

\section{OpenEdition}

\section{Journals}

Édition électronique

URL : http://journals.openedition.org/tvseries/1461

DOI : $10.4000 /$ tvseries. 1461

ISSN : 2266-0909

Éditeur

GRIC - Groupe de recherche Identités et Cultures

Référence électronique

Stéphane Thévenet, « La localisation à l'étranger dans les séries télévisuelles contemporaines et l'affirmation internationale de la Corée du Sud », TV/Series [En ligne], 2 | 2012, mis en ligne le 01 novembre 2012, consulté le 24 avril 2019. URL : http://journals.openedition.org/tvseries/1461 ; DOI : $10.4000 /$ tvseries. 1461

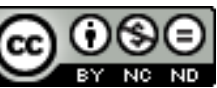

$T V /$ Series est mis à disposition selon les termes de la licence Creative Commons Attribution - Pas d'Utilisation Commerciale - Pas de Modification 4.0 International. 


\section{La localisation à l'étranger dans les séries télévisuelles contemporaines et l'affirmation internationale de la Corée du Sud

Très présents dans les intrigues des trendy dramas des années 90 mais peu représentés à l'écran jusqu'alors, les pays étrangers deviennent progressivement les lieux privilégiés de la rencontre amoureuse dans les miniséries à succès sud-coréennes du début des années 2000. Parfois espace de subversion, la terre étrangère devient aussi le lieu où est mise en scène, à travers les pérégrinations de ses citoyens, une Corée moderne et dynamique enfin reconnue internationalement, à l'image de ses grandes firmes internationales. La mégalopole de Séoul, avec son aéroport, point de départ et d'arrivée, en est le symbole. La localisation à l'étranger est cependant temporaire. À l'échelle du feuilleton, elle ne dure rarement plus de quelques épisodes. A partir des années 2005-2006, elle perd aussi de son importance diégétique. Les destinations touristiques de courte durée sont privilégiées.

$\mathrm{D}$ epuis la fin des années 1990, les dramas $^{1}$ coréens $^{2}$ (ou kdramas) sont au centre d'un engouement sans précédent appelé «Vague coréenne » ou Hallyu3. Apparu au milieu des années 90, ce large intérêt pour les produits de la culture populaire coréenne (musique, séries télévisées, cinéma), et en particulier pour les dramas, s'affirme à la fin des années 90 et atteint son apogée au début des années 2000. Il commence ensuite à s'essouffler à partir des années 2005-2006. Cette « vague » touche, principalement et dans un premier temps, la Chine continentale et méridionale et les pays de l'Asie du Sud-Est. Elle prend une plus grande ampleur en 2003 lorsqu'elle atteint le Japon, ancienne puissance colonisatrice et rivale économique, elle-même exportatrice de culture populaire. Même si la «Vague coréenne » semble actuellement avoir perdu de sa vigueur, les séries coréennes tiennent désormais, en Asie en tous les cas, la dragée haute aux séries venues du continent américain (séries américaines et telenovelas).

Bien que des formats plus longs et des thématiques différentes se soient parfois imposés (Jewel in the Palace 4 par exemple ${ }^{5}$ ), les

\footnotetext{
${ }^{1}$ Conformément à l'usage du terme en Corée et en Asie, drama désigne ici une série télévisée. Pour plus de commodité, le terme sera utilisé comme un nom commun de la langue française, c'est-à-dire avec les marques du pluriel lorsqu'elles s'imposent.

De même, les noms des personnes coréennes citées seront présentés selon l'usage en vigueur en Corée : nom (en majuscules) suivi du prénom.

${ }^{2}$ L'appellation " Corée » ainsi que les noms et adjectifs y faisant référence désigneront dans cet article la Corée du Sud. Lorsqu'il sera question de la partie nord de la péninsule, l'appellation complète « Corée du Nord » sera utilisée.

3 Le système de romanisation de la langue coréenne utilisé ici est celui mis en place par le gouvernement sud-coréen depuis juillet 2000.

4 대장금 [Daejang-geum] (MBC, 2003, 54 épisodes)

${ }^{5}$ Les titres des dramas seront traduits en français lorsque n'existent pas de titres officiels en anglais. Le titre original en coréen figurera en note de bas de page.
} 
" miniséries coréennes ${ }^{6}$ » au cœur de la «Vague coréenne » se présentent en général sous la forme de feuilletons d'une vingtaine d'épisodes ${ }^{7}$ diffusés en prime time (ce qui signifie, en Corée, 22 heures, après le journal télévisé). L'intrigue principale est construite autour d'une romance contemporaine mettant en scène de jeunes gens (de la fin de l'adolescence jusqu'à 35 ans environ) dans un environnement urbain. Dans le cadre d'une thèse de doctorat portant sur les séries coréennes récentes, un travail d'analyse des récurrences et des variations nous a permis de constater, à partir des années 2000, l'apparition de plus en plus fréquente de la localisation diégétique à l'étranger, accompagnée de sa mise en valeur dans les titres, les génériques et les extraits diffusés lors des campagnes promotionnelles. Ce phénomène, favorisé par l'augmentation des moyens de production et la popularisation des dramas coréens à l'étranger, est particulièrement marqué à partir de 2004: le nombre de dramas présentant une partie de leur intrigue à l'étranger passe alors de 2 à 12. Ce phénomène est net pendant trois ans mais se ralentit et se modifie ensuite $^{8}$. Il peut être interprété non seulement comme un désir d'exotisme et une tendance à la marchandisation des lieux de production mais également comme la manifestation d'une prise de confiance nationale qui se concrétise à l'écran par la mise en scène de la réussite des entreprises et des résidents coréens à l'étranger.

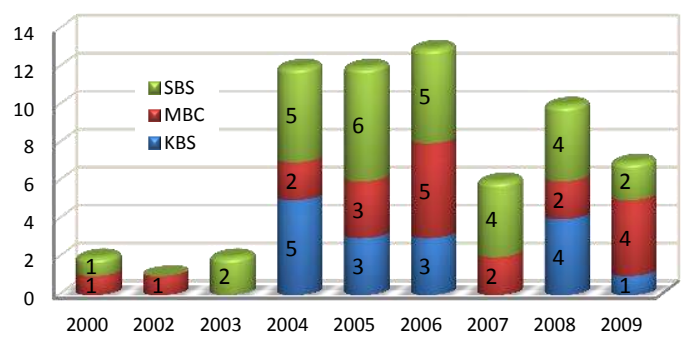

Fig. 1 : Evolution du nombre de dramas incluant une localisation diégétique à l'étranger (Données : Myung-hye Kim).

\footnotetext{
${ }^{6}$ L'appellation « minisérie » employée par les professionnels de la télévision coréenne est conservée ici mais, compte tenu du nombre d'épisodes, il s'agit en fait davantage de " feuilletons canoniques » ou parfois de " feuilletons sérialisants ». Voir Stéphane Benassi, "Sérialité(s)» in Sarah Sepulchre (dir.), Décoder les séries télévisées, De Boeck Editions, 2011, p. 84-89.

${ }^{7}$ Le système des « saisons » est quasi-inexistant en Corée.

8 Myung-hye Kim, "Visualizing the World Order in Korean television dramas », Colloque

IAMCR, Braga, Portugal, 18-22 juillet 2010.
} 
Le présent article, fondé sur l'analyse de dix miniséries à succès de la période 2003-2006, s'interrogera sur les raisons pour lesquelles, à une certaine période, la localisation à l'étranger est devenue un élément récurrent des scénarios. Il reviendra sur les spécificités narratives et génériques du drama coréen à succès qui permettent l'introduction dans la diégèse de la localisation à l'étranger, abordera le rôle prégnant que joue l'étranger dans l'imaginaire coréen et étudiera les rapports qu'entretiennent les personnages avec l'étranger, rapports qui semblent dessiner une nouvelle représentation de la Corée.

La « minisérie coréenne » est apparue à la fin des années 80 en Corée. Elle subit l'influence du trendy drama japonais qui émerge alors. Ciblant la classe émergente des jeunes consommateurs, le trendy drama - selon l'appellation utilisée par les chercheurs et les critiques asiatiques - relate les tribulations amoureuses de jeunes urbains exerçant généralement des professions dites « modernes » et attirantes (designer, journaliste, architecte, chanteur, etc.) qui leur permettent de disposer d'un bon niveau de vie et d'être ainsi à la pointe des dernières tendances en matière de vêtements, produits high-tech, meubles, voitures et autres biens de consommation. Le trendy drama est conçu comme un indicateur de tendances et le placement de produits qui le caractérise est favorisé par l'intrigue. Cette dernière se résume souvent en un récit amoureux qui s'ouvre avec la rencontre et se poursuit en atermoiements mettant en scène les différentes étapes heureuses et malheureuses de la relation amoureuse, avec le passage obligé que constitue la "fréquentation » (comme on l'appelait autrefois dans certaines régions françaises et en Suisse), c'est-à-dire la période de relation sentimentale qui précède généralement le mariage (yeonae en coréen ; dating en anglais). Le lieu de la rencontre ainsi que celui de la «fréquentation» contribuent fortement à l'esthétisation de la romance. Les scènes en extérieur, si possible proches de la nature, sont très appréciées ${ }^{9}$, mais la ville pensée comme un immense terrain de jeux consuméristes reste le cadre idéal des pérégrinations amoureuses.

À partir de 2000 avec, par exemple, All about Eve ${ }^{10}$ (où la destination est Londres), la localisation étrangère devient un terrain de plus en plus privilégié pour la rencontre amoureuse ou les retrouvailles (comme dans All In, SBS, 2003) tandis que la longue période de "fréquentation" qui suit trouve plus facilement sa place en Corée, pour des raisons très pragmatiques: il est ici question encore de

9 D'autant plus si la rencontre a lieu pendant l'enfance ou l'adolescence comme c'est le cas dans les très célèbres dramas de YOON Soek-ho, Autumn Tale (가을동화, KBS, 2000) et Winter Sonata (겨울연가, KBS, 2002) tournés dans des localités de province qui deviennent de véritables lieux touristiques fréquentés par les fans des séries.

${ }^{10} \mathrm{KBS}, 2000$. 
l'attrait des retombées économiques du placement de produits, plus facile à mettre en place dans un environnement urbain coréen. Les images d'une très grande qualité (nous sommes à l'époque de l'introduction de la qualité HD en Corée) révèlent Paris, Vienne, Prague, villes romantiques par excellence, et permettent une esthétisation maximale de ce moment féérique qui sera ensuite très souvent repris tout au long du feuilleton dans des séquences souvenirs accompagnées d'une ballade musicale. D'autres destinations moins attendues telles que Hong-Kong, Harvard, Beijing, Sydney et Bali jouent cependant le même rôle et permettent d'introduire une plus grande diversité. Surfant sur la "Vague coréenne ", les chaînes n'hésitent pas à investir dans un tournage à l'étranger, ce qui, dix ans plus tôt, serait apparu très risqué financièrement parlant et, une vingtaine d'années auparavant, totalement impensable. En effet, jusqu'à la fin des années 80 , les conditions économiques, sociales et surtout politiques ne permettaient nullement à la destination étrangère de trouver une place dans les représentations imaginaires collectives. Les intrigues restaient fortement ancrées dans la quotidienneté et le territoire national. À partir de la guerre de Corée (1950-1953) qui entérina la division de la péninsule en deux, la Corée du Sud subit trois décennies de dictature militaire limitant les libertés individuelles et notamment celle de voyager librement, sous prétexte de sécurité nationale. Il faut attendre la fin des années 80 pour que la démocratisation du pays se mette en marche et l'année 1989 pour que les restrictions de voyages soient entièrement levées.

Progressivement, la Corée du Sud, devenue une véritable puissance industrielle sous le joug des dictatures militaires, se tourne vers le monde extérieur et prend en marche le train de la mondialisation. À partir du milieu des années 90, l'étranger devient un élément narratif récurrent sans que l'on puisse voir pour autant les personnages évoluer ailleurs que sur le sol national. Les départs pour l'étranger et les retours en Corée n'étaient que très rarement prétexte à montrer des images d'autres pays. Ces départs étaient davantage conçus comme des astuces narratives qui permettaient aussi bien de séparer des amoureux, de désamorcer un conflit que de supprimer un personnage. Le plus souvent, l'émigrant quittait volontairement la Corée pour ses études, parfois pour travailler. Le personnage pouvait aussi être contraint à partir : le départ pour l'étranger ressemblait alors à une fuite ou à un exil forcé. À l'inverse, le retour d'un personnage après un long séjour à l'étranger était l'assurance de rebondissements. Quoi qu'il en soit, la caméra ne franchissait que très rarement les portes d'embarquement de l'aéroport et les spectateurs étaient ainsi les témoins de scènes de séparation (ou de retrouvailles), ingrédients privilégiés du genre mélodramatique qui gouverne les miniséries coréennes. Des scènes censées se dérouler à l'intérieur des avions en partance ou à destination de la Corée venaient aussi parfois illustrer 
ces moments particuliers dans la vie des héros. Les destinations étaient souvent les mêmes, avec une nette récurrence des États-Unis et de la France ${ }^{11}$. On se souvient, par exemple, du départ pour les États-Unis dans Autumn Tale ${ }^{12}$ (KBS2, 2000) et Winter Sonata ${ }^{13}$ (KBS, 2002) ou du départ pour la France dans First Love ${ }^{14}$ (KBS, 1997) et Romance ${ }^{15}$ (MBC, 2002).

En revanche, à partir de la fin des années 90 et surtout au début des années 2000, les téléspectateurs coréens assistent à l'apparition de séquences, voire d'épisodes entiers, qui se passent à l'étranger et qui non seulement tiennent une place importante dans l'histoire racontée mais participent aussi fortement à la mise en valeur du drama. La localisation est alors annoncée ou fortement suggérée dans le titre et mise en avant dans la bande-annonce ou les extraits proposés aux téléspectateurs. Ce phénomène débute timidement avec Stars in $M y$ Heart $^{16}$ (MBC, 1997) et All about Eve ${ }^{17}$ (MBC, 2000), deux miniséries coréennes typiques dont la forme narrative correspond à celle des trendy dramas. Il s'accentue considérablement à partir de 2003. Dix dramas qui ont été diffusés entre 2003 et 2006 et se sont particulièrement distingués par leur taux d'audience ont attiré notre attention. Il s'agit de :

All In (SBS, 2003, 24 ép.)

Paris Lovers (SBS, 2004, 20 ép.)

Sorry, I Love You (KBS2, 2004, 16 ép.)

Ireland (MBC, 2004, 16 ép.)

Love Story in Harvard (SBS, 2004-2005, 16 ép.)

Praha Lovers (SBS, 2005, 18 ép.)

What Happened in Bali (SBS, 2004, 20 ép.)

Beijing My Love (KBS2, 2004, 20 ép.)

Hong-Kong Express (SBS, 2005, 16 ép.)

Spring Waltz (KBS2, 2006, 20 ép.) ${ }^{18}$

\footnotetext{
${ }^{11}$ Il est intéressant de noter que, dans la langue coréenne, deux expressions spécifiques existent pour signifier le départ vers les États-Unis (domi) et le départ vers la France (dobul) mais qu'à l'exception de l'expression do-il qui fait référence au départ pour le Japon (vraisemblablement beaucoup moins courante), aucune autre destination ne semble avoir ce traitement de faveur.

12 가을동화 [ga-eul donghwa]

13 겨울연가 [ gyeo-ul yeon-ga]

14 첫사랑 [cheot sarang]

15 로망스 [romangseu]

16 별은 내 가슴에 [byeol-eun nae gaseume]

17 이브의 모든 것 [ibeu-e modeun geot]

${ }^{18}$ 올인 [orin], 파리의연인 [pari-ui yeonin], 미안하다 사랑한다 [mianhada sarang handa],

아일랜드 [a-illaendeu], 러브스토리인하버드 [reobeu seutori in habeodeu], 프라하의연인
} 
Trois d'entre eux ont des titres qui ne font pas référence à une localité étrangère (All In, Sorry, I Love You et Spring Waltz), mais ils mettent en avant les scènes tournées à l'étranger dans leur générique et dans leur premier épisode. En revanche, le drama intitulé Ireland n'offre paradoxalement aucune scène tournée en Irlande. Ainsi même les quelques scènes, très courtes, de souvenirs de Jung-a, fille d'origine coréenne adoptée par un couple d'Irlandais et « de retour» en Corée, ont en fait été tournées sur l'île coréenne de Jeju (dont le climat et les paysages ont peu de choses en commun avec l'Irlande, ce qui rend ces scènes peu réalistes).

Une représentation schématique des scènes censées se dérouler à l'étranger - qui prend en compte les séquences tournées en Corée en studio ou dans des lieux pouvant être perçus, avec ou sans artifice, comme des décors étrangers - permet de s'apercevoir que la place de l'étranger dans la diégèse, malgré sa mise en valeur, se cantonne en fait à quelques épisodes, les premiers en général. Seuls Beijing My Love, coproduction ${ }^{19}$ coréano-chinoise, et All In utilisent la localisation à l'étranger de façon plus importante (voir figure $2:$ les parties vertes indiquent les localisations à l'étranger).

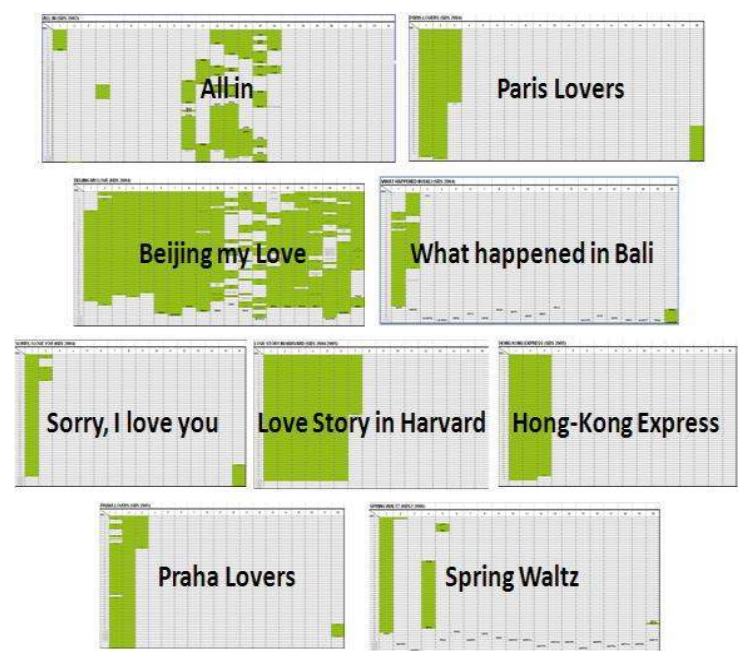

Fig. 2 : Représentation de la part des localisations à l'étranger (en vert) par rapport aux localisations en Corée (une colonne par épisode).

[peuraha-ui yeonin], 발리에서 생긴 일 [balli eseo saeng-gin il], 북경 내 사랑 [bukgyeong nae sarang], 홍콩 익스프레스 [hongkong ikseupeureseu], 봄의 왈츠 [bomui walcheu] ${ }^{19}$ En 2006, Tree of Heaven (SBS) une coproduction avec le Japon, favorisera, quant à elle, les localisations au Japon. 
Le premier à accorder une grande place au tournage étranger est All In, qui connaît un succès important en 2003 (35,7 \% de part d'audience ${ }^{20}$ ). Dans ce feuilleton, les quatre personnages principaux se rendent tour à tour aux États-Unis mais c'est surtout In-ha, le héros de la série, que l'on suit dans son exil. Il se réfugie aux États-Unis après avoir fui la Corée (épisode 9) et là, après avoir travaillé comme manutentionnaire dans un port (épisode 10), devient le garde du corps d'un milliardaire américain enrichi illégalement, puis devient champion de poker à Las Vegas (première scène du premier épisode qui laisse place ensuite à un long flash-back retraçant le parcours de inha). Les États-Unis sont également le lieu de retrouvailles avec la femme qu'il aime, venue poursuivre des études et peut-être aussi animée du vague espoir de retrouver In-ha. Ce drama offre de nombreuses scènes véritablement tournées sur place, notamment à Las Vegas, dans la région du Grand Canyon et sur un campus universitaire. Comme le montre KIM Myung-hye ${ }^{21}$, les Etats-Unis sont associés ici, comme dans de nombreux autres dramas, à la réussite personnelle. L'ascension sociale de In-ha, entré clandestinement sur le territoire américain, en est très représentative, et cela même si cet exemple déroge aux valeurs confucianistes peu enclines à approuver l'enrichissement par le jeu.

L'année suivante, Paris Lovers (2004) obtient plus de $50 \%$ de part d'audience en fin de diffusion ${ }^{22}$. Narrant la rencontre d'une étudiante coréenne qui vit chichement à Paris avec le fils du dirigeant d'une grande entreprise coréenne en passe de succéder à son père, ce drama prend les allures, par moments, d'un conte de fée moderne. Certaines scènes sont tournées dans les rues de Paris, à la Cité Internationale, au Jardin des Tuileries, mais également dans de belles demeures de la région parisienne et dans le Midi de la France. Cependant, dès le quatrième épisode, avec le retour de l'héroïne en Corée, la Seine et les bâtiments haussmanniens laissent presque définitivement place aux larges trottoirs des rues de Séoul et aux immeubles modernes.

${ }^{20}$ Voir Le livre blanc des audiences de télévision 2003, Rapport d'étude 2004, Korean Broadcasting Institute, Séoul, 2004, p. 169 [en coréen].

${ }^{21}$ Myung-hye Kim, "Visualizing the World Order in Korean television dramas », op. cit. Rappel: le nom des personnes coréennes citées (sauf références bibliographiques) est présenté selon les normes en usage en Corée : NOM (en capitales) suivi du prénom.

${ }^{22}$ Sa part d'audience atteint $57,9 \%$ le 15 août 2004. 


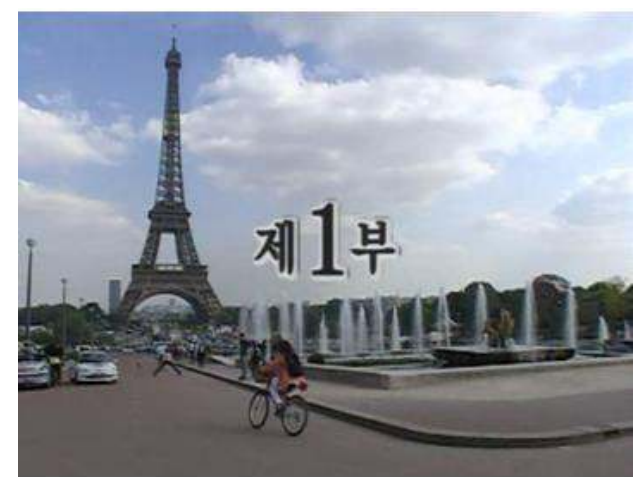

Fig. 3 : Paris Lovers, épisode 1

Le pays étranger s'affirme comme le lieu de prédilection de la rencontre amoureuse. L'exotisme de la rencontre reste cependant décoratif : de l'autre côté de la Mer Jaune ou à plusieurs milliers de kilomètres de Séoul, les Coréens finissent invariablement par rencontrer un de leurs concitoyens (Lovers in Paris, What Happened in Bali, All In, Praha Lovers) ou des autochtones physiquement et culturellement très proches qui parlent le coréen - Yang-sue, étudiante chinoise, dans Beijing My Love; Mu-hyeok, petit malfrat australien d'origine coréenne dans Sorry, I Love You.

La terre étrangère peut aussi, parfois, représenter un espace de transgression ou, tout du moins, un espace de liberté. Loin de la société coréenne et du poids des traditions, le personnage peut déroger plus facilement non seulement aux lois en vigueur en Corée mais également aux lois tacites. Il semble ainsi plus acceptable de voir sur le sol étranger un Coréen posséder ou utiliser une arme à feu ou bien être blessé voire tué par une arme.

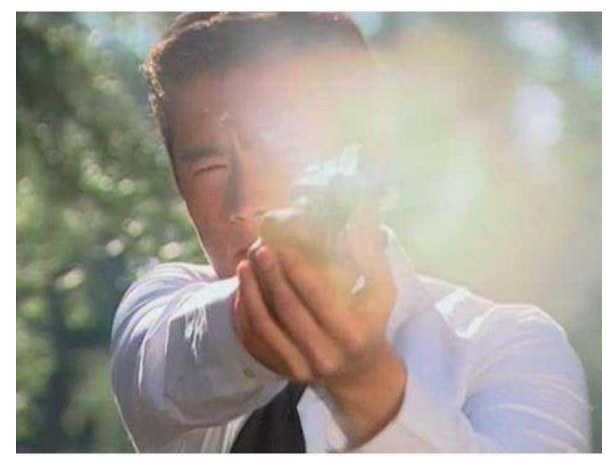

Fig. 4 : In-ha en action dans All In 
Interdites en Corée, les armes à feu n'apparaissent que très rarement, voire jamais, entre les mains de simples individus - seule la police est armée - dans les dramas se déroulant en Corée. Cependant, dans trois dramas du corpus d'observation, un ou plusieurs personnages principaux sont tués ou gravement blessés par balle alors qu'ils sont à l'étranger. C'est le cas de Mu-hyeok dans Sorry, I Love You, de In-ha dans All In et de Su-jeong et In-uk dans What Happened in Bali. De même, la représentation dans un même lit d'un couple vedette ${ }^{23}$ est envisageable à l'étranger mais difficilement en Corée ${ }^{24}$. Dans l'épisode 13 de All In, les téléspectateurs peuvent ainsi voir In-ha allongé dans les bras de Su-yeon, l'embrasser tendrement et s'apprêter à déboutonner son chemisier puis, dans la scène suivante, retrouver Su-yeon, le lendemain matin, apparemment nue sous les draps. De la même façon, dans le dernier épisode de What Happened in Bali, les téléspectateurs partagent les derniers instants de Su-jeong et In-uk, tués par balle alors qu'ils se trouvent allongés l'un à côté de l'autre, nus sous les draps. Enfin, l'accès aux casinos, interdit aux Coréens en tant que clients, sur le territoire national, devient possible à Las Vegas (All In).

Ces quelques exemples présentent la terre étrangère comme un espace éventuel de subversion et invitent à s'attarder sur le rapport ambigu que la Corée entretient avec l'étranger. Située à l'extrémité sud de la péninsule coréenne et séparée du reste du continent par une frontière quasiment imperméable partagée avec la Corée du Nord, la Corée peut être, en pratique, assimilée à une île que l'on ne peut quitter ou rejoindre que par avion ${ }^{25}$ ou par bateau ${ }^{26}$. Pourtant, malgré l'ouverture tardive du pays, les échanges avec l'étranger sont multiples et variés et les citoyens coréens sont présents en grand nombre sur tous les continents.

Dans un contexte d'hyper concurrence entre les trois chaînes généralistes (KBS, MBC, SBS ${ }^{27}$ ) qui dominent la production et le marché, l'immense succès des premiers dramas mettant en avant la

\footnotetext{
${ }^{23}$ Les personnages masculins qui s'inscrivent en opposition au personnage masculin principal sont présentés plus facilement dans ce genre de situation (sans qu'aucun acte sexuel ne soit montré, tout juste suggéré).

${ }^{24}$ Les mentalités commencent, cependant, à changer. En 2005, My Name Is Kim Samsun (MBC), succès d'audience, mettait en scène, à plusieurs reprises, l'héroïne principale dans un lit avec l'homme qu'elle aimait et l'intrigue suggérait la suite des événements, invisibles pour les téléspectateurs.

${ }^{25}$ Les compagnies aériennes nationales (Korean Air ou Asiana Airlines) sont facilement identifiables dans les dramas: une vue de l'avion qui transporte les personnages en partance de, ou à destination de, la Corée est très souvent donnée à voir. De plus, les compagnies aériennes apparaissent presque systématiquement dans la liste des sponsors. ${ }^{26}$ Très rarement représenté à l'écran. Dans All In, cependant, In-ha et Chong-gu embarquent clandestinement dans un cargo.

${ }^{27}$ KBS : Korean Broadcasting System ; MBC : Munhwa Broadcasting System ; SBS : Seoul Broadcasting System.
} 
localisation à l'étranger est sans conteste la raison principale de la ruée - temporaire - des équipes de production vers les aéroports. Néanmoins, nous pouvons aussi voir, à travers cette émergence de la localisation à l'étranger, une façon de marquer l'entrée sur la scène internationale de la Corée. La «Vague Coréenne » est un phénomène sans précédent qui a fortement marqué les esprits. Elle se forme à la fin des années 90 à un moment important de l'accès à la visibilité internationale de la Corée du Sud. La réussite économique et la démocratisation l'ont fait connaître au monde. Différents événements de nature et de portée différentes la placent directement sous les feux de la rampe. À la veille du second millénaire, la Corée, qui a souffert jusqu'alors d'un manque de reconnaissance internationale, accède à un certain rang mondial et en prend conscience.

Les principaux événements qui la précipitent sur le devant de la scène internationale sont les suivants : la crise économique asiatique de 1997 a été dévastatrice pour la société et l'économie des pays asiatiques en général mais la Corée du Sud se redresse rapidement malgré les plans de rigueur imposés par le FMI et les répercussions sur l'économie nationale. Elle est félicitée alors pour son exemplarité. Quelques années plus tard, en 2000, le prix Nobel de la paix est remis au président Kim Dae-jung, récompensé pour son action en faveur de la démocratie et du rapprochement avec le Nord. La Coupe du monde de football de 2002, organisée conjointement par la Corée et le Japon, ancien ennemi, est également très positive pour l'image de la Corée. Elle accède, à la surprise générale, aux quarts de finale tandis que l'enthousiasme de ses supporters devient célèbre dans le monde entier. Le succès des produits culturels coréens en Asie et, dans une moindre mesure, dans certaines parties du globe, conforte la Corée dans son désir de reconnaissance internationale. CHO Hae-joang, notamment, montre que l'engouement pour la culture populaire coréenne en Asie a engendré de nombreux discours nationalistes, culturalistes et capitalistes tenus aussi bien au niveau académique, médiatique que gouvernemental ${ }^{28}$.

Les dramas coréens qui accordent une part importante à la localisation étrangère sont avant tout l'occasion de montrer, à travers les entreprises coréennes implantées sur le sol étranger et les citoyens coréens expatriés ou en séjour à l'étranger, un pays qui s'affirme sur la scène internationale. Samsung, LG, Daewoo, Hyundai : voici des marques coréennes que tout un chacun côtoie quotidiennement, ne serait-ce qu'en actionnant son téléphone portable, son téléviseur ou son ordinateur. Ces marques s'affichent en grand sur les murs et les toits des capitales du monde. Grandes entreprises familiales (conglomérats ou jaebeol en coréen), elles occupent également une

${ }^{28}$ Hae-joang Cho, « Reading the Korean Wave as a Sign of Global Shift », Korea Journal, vol. $45, \mathrm{n}^{\circ} 4$, Winter 2005, p. 147-182. 
place importante dans la société coréenne et dans l'estime de beaucoup de Coréens. Figurant parmi les principaux sponsors, elles sont présentes depuis longtemps à l'écran. La Corée a, en effet, développé dans les années 80-90 un genre de feuilleton surnommé "dramas d'entreprise » (gi-eop drama) dont le cadre principal est une firme et qui met en scène des personnages dirigeants et salariés. Les dramas à la base de notre étude, en donnant à voir les grandes compagnies coréennes à l'étranger à travers des personnages principaux dirigeants ou fils de dirigeants, apparaissent comme une réminiscence de ce genre. Cet aspect est flagrant dans quatre des dramas présentés plus haut: Paris Lovers, Beijing My Love, Hong-Kong Express et What Happened in Bali. Présentes à Paris, Beijing, Hong-Kong et Jakarta, les puissantes entreprises coréennes contribuent à montrer la Corée comme acteur important de la mondialisation. Dans Paris Lovers (2004) notamment, le parallèle que l'on peut déceler entre l'entreprise fictive GD et l'entreprise GMD, née du rapprochement (en 2002) entre Daewoo et General Motors, est d'ailleurs troublant (voir figures 5 et 6). Le logo de l'entreprise fictionnelle, elle aussi productrice d'automobiles, très souvent présent en arrière-plan ou dans des détails récurrents (pins de l'entreprise portés sur les vêtements, sigle des véhicules ...), comporte de nombreuses similitudes avec celui de l'entreprise réelle. En cela, l'entreprise fictive GD non seulement participe à la construction médiatique d'une Corée puissante économiquement parlant mais elle permet aussi à GMD d'assurer sa promotion sur la scène nationale et internationale. La pratique peut donc être assimilée à du placement de produits et l'idéologie sousjacente à du nationalisme économique.

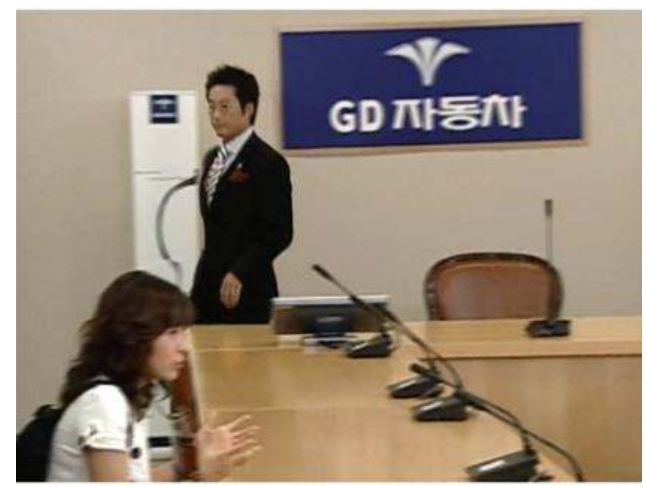

Fig. 5 : Paris Lovers (épisode 2) : logo de GD Jadongcha (=cars) 


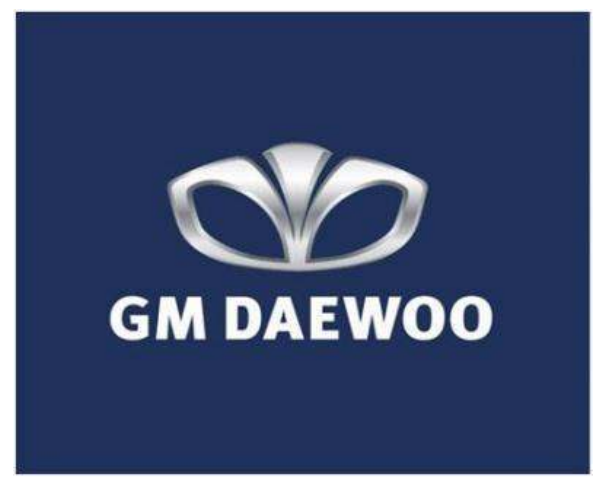

Fig. 6 : Logo de General Motors Daewoo.

L'image de la Corée à l'étranger est une question sensible pour beaucoup de Coréens. Elle est très dépendante du comportement et de la réussite de leurs concitoyens présents à l'étranger temporairement ou de façon permanente, voire jusqu'aux membres de la diaspora coréenne. Les dramas de notre corpus mettent ainsi en scène à l'étranger des personnages puissants et ambitieux. Chefs d'entreprise, stars de la chanson, champion du monde de poker, musicien célèbre, diplomate, ils disposent de revenus conséquents qui leur permettent d'avoir de très bonnes conditions de vie, supérieures à celles de la majorité des autochtones. L'arrogance et l'outrecuidance de Gisu, jeune dirigeant d'entreprise dans Paris Lovers, sont ainsi très représentatives. Au cours du premier épisode, alors qu'il remporte une partie de poker organisée dans une somptueuse villa de la Côte d'Azur, il n'hésite pas à jeter dans la piscine le contenu de la mallette de billets qu'il vient de gagner, sous les yeux médusés des Français. Dans Love Story in Harvard, la séquence de la fête de bienvenue aux nouveaux étudiants de la Law School est aussi significative. Les étudiants sont rassemblés devant un bâtiment et deux personnes apparaissent au balcon qui les surplombe. La première personne est une jeune femme coréenne qui formule un discours remarquable. Ensuite, un homme plus âgé, également asiatique, prend la parole à son tour et se présente comme l'organisateur de la soirée. Les téléspectateurs apprennent alors par la bouche des étudiants médusés que ces deux personnes sont en fait un avocat célèbre enrichi grâce à son succès, et sa fille. Tous les étudiants, américains et étrangers, regardent et écoutent avec envie et admiration ces deux Coréens, symboles de réussite sociale et professionnelle. Plus loin dans l'épisode, dans cette même Law School, la classe d'un professeur illustre mais non moins autoritaire devient le terrain d'affrontement entre Hyeon-u et Jeong-min, les deux plus talentueux élèves de la classe, qui n'ont de cesse de montrer leur connaissance et leur intelligence et ont la particularité d'être coréens. 
Dans Hong-Kong Express, la puissance du PDG du Groupe multinational JR est telle que tous les employés de l'hôtel international où il est descendu font preuve d'une très grande déférence, le suivant constamment pour répondre à ses moindres volontés. La première séquence de All In montre In-ha à Las Vegas remportant triomphalement le championnat du monde de poker, devançant tous les meilleurs joueurs mondiaux. Les exemples tirés de notre corpus où un héros coréen est mis dans une situation de supériorité pourraient être ainsi multipliés. Lorsque les personnages sont de simples étudiants, leur ambition s'exprime avant tout à l'échelle internationale: ainsi, Hyeon-u (Love Story in Harvard), dès son arrivée à Harvard, affiche le drapeau coréen au mur de sa chambre (voir figure 7) et clame son désir, en tant que représentant du peuple coréen, de rentrer en compétition avec les autres étudiants américains.

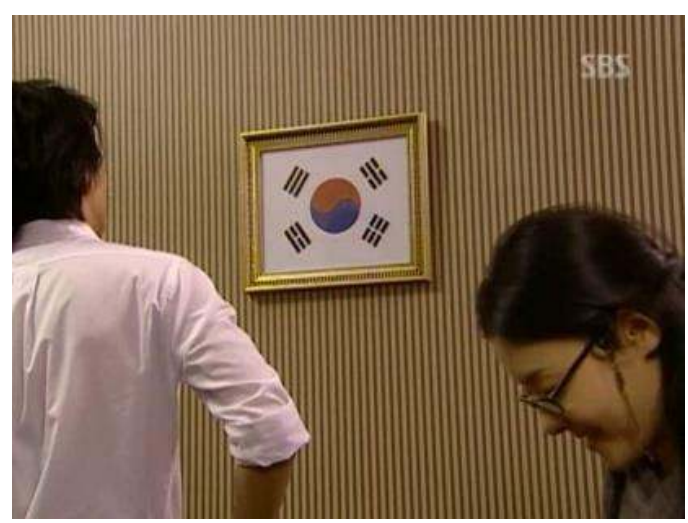

Fig. 7: Love Story in Harvard, épisode 1

Cependant, la facilité avec laquelle les personnages coréens trouvent leur place dans la mondialisation se manifeste aussi à travers la grande habilité qui leur est prêtée à parler les langues étrangères : anglais, chinois, allemand, indonésien, tchèque, la langue de la contrée d'accueil est toujours parlée par au moins un des personnages coréens. Certains personnages, telle Su-yeon dans All In en maitrisent plusieurs (japonais et anglais).

Comme nous l'avons souligné précédemment, aucune intrigue n'est exclusivement située à l'étranger. Une fois l'élu de leur cour rencontré, les hasards de la vie ramènent inexorablement les héros dans leur patrie d'origine. De retour, le personnage retrouve ses habitudes dans son pays et sa ville et le drama peut apparaitre alors, sous certains aspects, comme un outil de promotion de la capitale internationale qu'est Séoul, métropole de plus de dix millions d'habitants qui centralise tous les pouvoirs. À travers les dramas de la 
période qui privilégient les localisations à l'étranger, les téléspectateurs ont découvert ou redécouvert des espaces urbains qui, à un moment ou à un autre, se retrouvent juxtaposés aux paysages de Séoul. La ville elle-même est, depuis longtemps, lieu de tournage - de nombreuses scènes des dramas sont ainsi tournées dans ses rues, parcs d'attractions, galeries d'art, cafés et restaurants ${ }^{29}$, transport en commun (bus et métro) et avant tout ses gratte-ciel ; elle en ressort magnifiée, ou tout du moins auréolée d'une image de ville moderne et dynamique. Ceci est flagrant dans le drama Lovers in Praha (voir figure 8), qui participe grandement à la représentation idéalisée de la Corée que peuvent se faire les téléspectateurs asiatiques consommateurs des produits de la «Vague coréenne », notamment ceux issus des pays de l'Asie du Sud-Est.

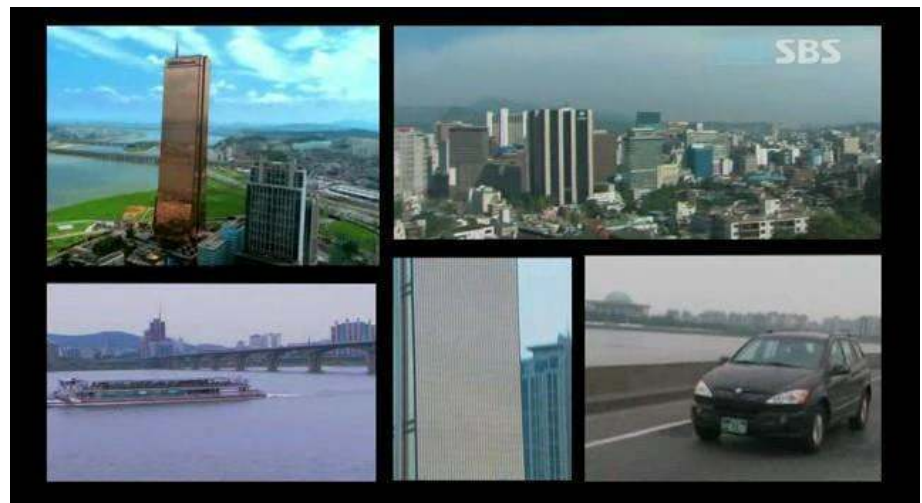

Fig. 8 : Lovers in Praha (épisode 3). Les images de la ville de Prague disposées selon le même agencement laissent la place, une à une, à celles de la ville de Séoul marquant ainsi le « retour » de l'intrigue en Corée.

Un endroit emblématique est, sans aucun doute, l'aéroport international d'Incheon, situé sur une île proche des côtes, plaque tournante des liaisons aériennes en Asie et dans le monde. Primé à plusieurs reprises parmi les meilleurs aéroports du monde ${ }^{30}$, il est devenu un élément important des dramas depuis son ouverture en 2001. Son architecture et ses équipements modernes y apparaissent fréquemment et, en 2007, il est même devenu le cadre d'un drama entier, Air City (KBS).

Alors que la « Vague coréenne » perd en vitalité, que le concept commence à s'user, et devant la nécessité de réduire les coûts de

${ }^{29}$ Les grands hôtels internationaux, nombreux à Séoul, sont aussi des lieux de tournage privilégiés. Ils apparaissent très fréquemment parmi les sponsors.

${ }^{30}$ L'aéroport est premier du classement World Airport Awards en 2009, troisième en 2011. 
production en temps de crise, les localisations à l'étranger tendent à diminuer à partir de 2006. Il s'agit d'une diminution en durée, mais aussi une diminution en importance narrative. Alors qu'elles étaient, entre 2003 et 2006, le lieu privilégié de rencontres amoureuses entre Coréens résidant au moins temporairement à l'étranger, les séquences qui se passent désormais à l'étranger n'ont pas d'autre valeur diégétique que d'être des destinations agréables pour des voyages d'affaire, des voyages entre amis ou entre amoureux ${ }^{31}$. C'est notamment le cas dans Palace ${ }^{32}$ (où la destination est la Thaïlande) en 2006 et dans Boys over Flowers 33 (Macao et Nouvelle-Calédonie) en 2009. Le plaisir visuel et l'exotisme y sont avant tout recherchés. Des changements s'opèrent, en conséquence, au niveau du sponsoring avec une recrudescence des agences de voyage et des offices de tourisme étrangers comme sponsors. Le drama On Air 34 (SBS, 2008) - dont le scénariste KIM Eun-sook et le réalisateur SIN Woo-cheol, sont aussi ceux de Paris Lovers et Praha Lovers - qui dépeint le milieu de la fiction télévisée montre, à travers les échanges entre la scénariste vedette et le producteur lors de la préparation de leur futur drama, à quel point la localisation à l'étranger semble être devenue un élément obligatoire, mais sans réelle importance diégétique. Le producteur cède ainsi aux pressions exercées par une agence de promotion taïwanaise et organise un tournage à Jiufen de manière tout à fait inopinée.

La dynamique entre répétition et variation au cœur de la série télévisuelle fait de cet objet d'étude un précieux indicateur de son époque de production. En tant que résultat d'un « compromis provisoire 35 », elle incorpore les conventions les plus massivement répandues tout en restant sensible aux courants d'innovation. Le drama coréen des années 2000, digne héritier du trendy drama, participe à la mode des localisations à l'étranger tout en conservant ses traditions mélodramatiques et perpétuant ses clichés. L'étape suivante, qui survient dès les années 2005-2006, est l'introduction de visages étrangers. L'exemple le plus connu est l'acteur coréano-américain Daniel Henney apparu dans My Name Is Kim Samsun (MBC, 2005).

${ }^{31}$ On note quelques exceptions, cependant, comme dans Iris (KBS, 2009) où la localisation en Hongrie s'explique par un projet d'attentat à l'encontre d'un dignitaire nord-coréen.

$3^{2}$ 궁 gung (MBC, 2006)

33 꽃보다남자 kkotboda namja (KBS, 2009)

34 온에어

35 Eric Macé, «Qu'est-ce qu'une sociologie de la télévision? (2) esquisse d'une théorie des rapports sociaux médiatisés. Les trois moments de la configuration médiatique de la réalité : production, usages, représentations » in Réseaux nº 105, 2001, p. 205 


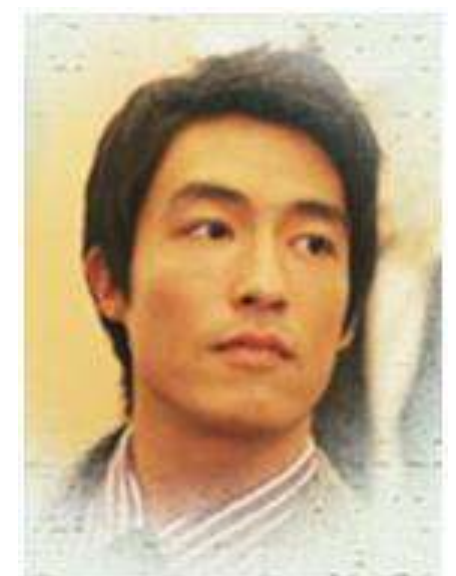

Fig. 9 : L’acteur coréano-américain Daniel Henney

L'exotisme est toutefois limité : ces nouveaux visages étrangers sont soit ceux d'Asiatiques avec une bonne connaissance de la langue coréenne soit ceux d'Occidentaux avec une filiation coréenne et donc avec un visage plutôt asiatique. Tout comme les localisations à l'étranger quelques années auparavant, ils introduisent un peu d'exotisme dans une narration qui demeure proche de celle d'une comédie romantique. Dans le monde globalisé d'aujourd'hui, le récit des miniséries coréennes reste celui de rencontres amoureuses entre Coréens ou assimilés. Comme le décrit Arjun Appadurai, l'élan vers la mondialisation de la Corée prend la forme d'une «production globale de la localité $3^{6}$ ». S'inscrivant dans une politique de reconstruction de la «coréanité », les diasporas coréennes 37 , voire les populations d'adoptés coréens ${ }^{38}$, sont englobées dans le nouveau monde coréen. De la même façon, après avoir, pendant des décennies, tourné le dos à ses proches voisins (Chine, Japon, dans un premier temps, les autres pays asiatiques ensuite), la Corée les redécouvre notamment dans le cadre des échanges suscités par la «Vague Coréenne». La question de la représentation fictionnelle des « autres Coréens » de l'autre côté du $38^{\mathrm{e}}$ parallèle, dans le genre de drama qui nous intéresse, reste, elle, posée.

${ }^{36}$ Arjun Appadurai, Après le colonialisme, les conséquences culturelles de la globalisation, Paris, Petite Bibliothèque Payot, 2005, p. 270.

37 Voir Eun-sil Yim, «Confrontations Nord/Sud au-delà de la péninsule : les Coréens du Kazakhstan " in Valérie Gelézeau (dir.), "Coopérations coréennes ", Critique internationale $\mathrm{n}^{\circ}$ 49, Paris, Presses de Sciences Po, 2010.

$3^{8}$ Voir Elise Prébin, «Adoption internationale : les revenants de Corée », Thèse de doctorat en ethnologie et sociologie comparative, Université de Paris X, Paris, 2006. 


\section{Bibliographie}

APPADURAi Arjun, Après le colonialisme, les conséquences culturelles de la globalisation, Paris, Petite Bibliothèque Payot, 2005.

CHо Hae-joang, « Reading the Korean Wave as a Sign of Global Shift », Korea Journal, vol. 45, nº 4, Winter 2005, p. 147-182.

GELEZEAU Valérie, «Espaces d'ailleurs, théâtres de prestige, Grands hôtels et sociabilités séouliennes » in Sanjuan Thierry (dir.) Les grands hôtels en Asie, modernité, dynamiques urbaines et sociabilité, Paris, Publications de la Sorbonne, 2003, p. 191-205.

KIM Myung-hye, «Visualizing the World Order in Korean television dramas », Colloque IAMCR, Braga, Portugal, 18-22 juillet 2010.

Korean Broadcasting Institute, Le livre blanc des audiences de télévision 2003, Rapport d'étude 2004, Séoul, 2004. [en coréen].

MACE Eric, « Qu'est-ce qu'une sociologie de la télévision ? (2) esquisse d'une théorie des rapports sociaux médiatisés. Les trois moments de la configuration médiatique de la réalité : production, usages, représentations » in Réseaux $\mathrm{n}^{\circ} 105,2001$, p. 199-242.

Prebin Elise, "Adoption internationale : les revenants de Corée », Thèse de doctorat en ethnologie et sociologie comparative, Université de Paris X, Paris, 2006.

YIM Eun-sil, «Confrontations Nord/Sud au-delà de la péninsule : les Coréens du Kazakhstan » in Valérie GELEZEAU (dir.), « Coopérations coréennes ", Critique internationale ${ }^{\circ} 49$, Paris, Presses de Sciences Po, 2010. 